\title{
Differences in Physical and Psychosocial Characteristics Between CFS and Fatigued Non-CFS Patients, a Case-Control Study
}

\author{
Veronique De Gucht $^{1}$ • Franshelis Katerinee Garcia ${ }^{1}$ - Marielle den Engelsman ${ }^{1}$. \\ Stan Maes ${ }^{1}$
}

Published online: 19 February 2016

(C) The Author(s) 2016. This article is published with open access at Springerlink.com

\begin{abstract}
Purpose The main research question is: "Do CFS patients differ from fatigued non-CFS patients with respect to physical, cognitive, behavioral, social, and emotional determinants?" In addition, group differences in relevant outcomes were explored.

Method Patients who met the Centers for Disease Control (CDC) criteria for CFS were categorized as CFS; these patients were mainly recruited via a large Dutch patient organization. Primary care patients who were fatigued for at least 1 month and up to 2 years but did not meet the CDC criteria were classified as fatigued non-CFS patients. Both groups were matched by age and gender ( $N=192$ for each group). Results CFS patients attributed their fatigue more frequently to external causes, reported a worse physical functioning, more medical visits, and a lower employment rate. The results of a multiple logistic regression analysis showed that patients who believe that their fatigue is associated with more severe consequences, that their fatigue will last longer and is responsible for more additional symptoms are more likely to be classified as CFS, while patients who are more physically active and have higher levels of "all or nothing behavior" are less likely to be classified as having CFS.

Conclusion A longitudinal study should explore the predictive value of the above factors for the transition from medically unexplained fatigue to CFS in order to develop targeted
\end{abstract}

Veronique De Gucht

degucht@fsw.leidenuniv.nl

1 Health, Medical, and Neuropsychology Unit, Leiden University, Wassenaarseweg 52, P.O. BOX 955, 2300

RB Leiden, The Netherlands interventions for primary care patients with short-term fatigue complaints.

Keywords Chronic fatigue syndrome $\cdot$ Fatigue $\cdot$ Matched case-control $\cdot$ Illness representations $\cdot$ Behavioral regulation pattern $\cdot$ Psychosocial characteristics

\section{Introduction}

Fatigue can be defined as "a sense of physical tiredness and lack of energy, different from sadness and weakness" [1]. About 18 to $39 \%$ of the general adult population experiences fatigue [2-5]. Fatigue can be seen as a continuum, ranging from mild complaints to severe disabling and persisting fatigue, like chronic fatigue syndrome (CFS) [6].

CFS is a chronic condition that is, according to the Centers for Disease Control (CDC) criteria, characterized by unexplained, severe, and persistent fatigue lasting for at least 6 months; the fatigue is not due to ongoing exertion or to a medical condition, is not improved by rest and may be worsened by physical or mental exertion, significantly interferes with daily activities and work, and is accompanied by four or more out of eight other somatic symptoms [7]. The prognosis of CFS is often poor with less than $10 \%$ returning to premorbid levels of functioning if the condition is left untreated [8].

Several studies have shown that CFS patients differ from fatigued non-CFS patients [9-11]. Fatigue in CFS patients is often more severe than in fatigued non-CFS patients, and CFS patients report more somatic symptoms. CFS patients more frequently believe that their fatigue will last for a very long time and will be associated with more adverse consequences, but CFS patients and fatigued non-CFS patients do not seem to differ in perceptions of control over their fatigue [9]. 
Furthermore, studies have shown that CFS patients attribute their illness rather to external physical causes than to internal or psychological causes $[12,13]$, and their levels of psychological distress are higher [9-11]. In terms of outcomes, CFS patients are found to be less able to conduct everyday activities, their level of physical functioning is lower, they visit more frequently their general practitioner (GP), have a higher absenteeism from work, and a lower employment rate [9-11]. Each of the above studies has, however, included only a limited set of determinants and varied importantly in terms of the comparison group that was used, depending upon the research question.

As it proves to be very difficult to influence CFS once it is firmly established, efforts should be directed at interventions at an earlier stage, targeting determinants that discriminate significantly between a non-CFS group and a CFS group, as these factors could also be responsible for a transition from short-term fatigue to CFS. The present study aims at identifying such determinants. The broad range of physical, cognitive, behavioral, social, and emotional determinants included in the present study was selected based on reviews of factors that may play a role in the development and maintenance of CFS [14-17] and the comparative studies cited above [9-13].

The present study is a matched case-control study including a broad set of modifiable determinants and some relevant outcomes. The central research question is: Do CFS patients, who meet the CDC criteria, differ from fatigued non-CFS patients, reporting fatigue for at least 1 month up to 2 years, with respect to physical (sleep, physical activity), cognitive (illness representations), behavioral (all or nothing and limiting behavior), social (social support), and emotional determinants (anxiety, depression)? In addition, group differences in a number of relevant outcomes (physical functioning, work status, and medical visits) were explored.

\section{Methods}

\section{Participants and Procedure}

CFS patients were recruited from a large Dutch patient organization and filled in an online questionnaire. Patients reporting fatigue of at least 1-month (but less than 2 years) duration were recruited via primary health care centers and completed the same online questionnaire. All participants signed an informed consent before taking part in the study. Participants who had a concurrent somatic condition that could explain the fatigue symptoms or those who had a severe psychiatric disorder were excluded from the study. The inclusion or exclusion was done on the basis of self-report.

A total of 723 patients completed the questionnaire. Onehundred and twenty-one participants were excluded due to missing data and 39 due to the presence of a somatic condition and/or psychiatric disorder. The final sample consisted of 563 participants. Participants were then categorized as CFS cases if they met the CDC criteria for CFS. Participants who reported fatigue for at least 1 month but did not meet the CDC criteria for CFS were categorized as fatigued non-CFS cases. The CDC criteria were assessed on the basis of a self-report questionnaire [18]. A total of 267 were categorized as CFS cases (202 of the patient organization and 65 of the primary care group) and 296 as fatigued non-CFS cases (all from the primary care group). Both groups were subsequently matched by age and gender using the SPSS plugin FUZZY. The final matched sample consisted of 192 CFS and 192 fatigued nonCFS cases. The mean age of the CFS group was 40.50 $(\mathrm{SD}=12.20)$, and the mean age of the fatigued non-CFS group was $40.22(\mathrm{SD}=12.20)$. Both groups consisted of 22 males $(11.5 \%)$ and 170 females (88.5\%).

\section{Measures}

\section{Patient Characteristics Include Age, Gender, and Educational Level}

\section{Fatigue and Somatic Complaints}

Fatigue severity was assessed using the subjective experience of fatigue subscale of the Checklist Individual Strength (CIS20R) [19] with higher scores indicating more fatigue.

Fatigue duration was assessed by means of self-report (number of weeks/months fatigued).

Somatic complaints were assessed with the Patient Health Questionnaire-15 [20]. Higher scores indicate more somatic complaints.

\section{Determinants}

Sleep was measured by asking participants to indicate the average amount of hours they sleep per night.

Physical activity levels were measured using the Short Questionnaire to Asses Health Enhancing Physical Activity [21]. Higher scores indicate more physical activity.

Patients' illness representations were assessed using the Brief Illness Perception Questionnaire (Brief IPQ) [22]. Higher scores on the dimensions consequences, timeline, identity, and emotional representation indicate more severe consequences, a longer expected fatigue duration, more additional symptoms, and more emotional consequences. Higher scores on personal control, treatment control, and understanding indicate higher beliefs in personal or treatment control and more understanding of one's fatigue. For the cause dimension, patients were given a list of nine potential causes and had to indicate for each of these causes if they considered it to be a 
potential cause of their fatigue/CFS or not (making use of a yes/no scale).

Behavioral regulation patterns were measured using the "all or nothing" (a pattern of over-activity followed by rest) and "limiting behavior" (reducing daily activities and excessive resting) scales of the Behavioral Reponses to Illness Questionnaire (BRIQ) [23]. Higher scores indicate more "all or nothing" or "limiting behavior."

Social support was measured with two questions: "How much social support do you receive from your partner (best friend or family member) for your fatigue?" and "How much social support do you receive from others who are not your partner (best friend or family member)?," rated on a 5-point Likert scale ranging from 'no support' to "a lot of support."

Psychological distress was assessed with the anxiety and depression subscales of the Brief Symptom Inventory (BSI) [24].

\section{Outcome Variables}

Physical functioning was assessed using the Short Form Health Survey (SF-12 v2) [25]. Lower scores indicate worse physical functioning.

Employment status and medical visits during the past 6 months were assessed by self-report.

\section{Statistical Analysis}

First, comparisons between the CFS and the fatigued non-CFS group were made using independent $t$ tests for normally distributed continuous variables, Mann-Whitney $U$ tests for nonnormally distributed continuous variables, and $\chi 2$ for categorical variables. To reduce the chances of obtaining falsepositive results, due to multiple comparisons, a Bonferroni correction was used. The Bonferroni-corrected alpha level that corresponds with an alpha level of 0.05 was $0.002(0.05 / 30)$. All $p$ values lower or equal to 0.002 were considered to be significant. All tests performed were two-tailed tests.

Next, a multivariate logistic regression analysis was conducted to identify which determinants contributed most to the likelihood of being diagnosed with CFS. The analyses were conducted using SPSS v21 for windows.

\section{Results}

Table 1 shows the results of the comparative tests. With regard to patient characteristics, no significant differences were found for age or gender, as subjects were matched for both age and gender. No significant difference was found for educational level. CFS patients reported a significantly longer duration of fatigue, a higher fatigue severity, and more somatic complaints than fatigued non-CFS patients.
In terms of physical, cognitive, behavioral, social, and emotional determinants, univariate differences were found for average hours of sleep per night, minutes of physical activity per week, perceived consequences, timeline, treatment control and identity (illness beliefs), "limiting" and "all or nothing behavior.". Non-CFS patients more frequently attributed their fatigue to "stress" or "their own behavior" (internal causes) than CFS patients, while CFS patients more frequently attributed their fatigue to "a germ or virus," "chance," and "poor medical care in the past" (external and physical causes). No significant differences were found for social support, anxiety, and depression.

With respect to relevant outcomes, the fatigued non-CFS group reported a significantly better physical functioning, a higher employment rate, and less medical visits.

Next, a multivariate logistic regression analysis was conducted to identify which determinants contribute most to the likelihood of being diagnosed with CFS. Only determinants that were significant at a univariate level (see Table 1) were entered as independent variables into the equation. Fatigue duration, fatigue severity, and somatic complaints were not entered as control or independent variables in the equation because they were part of the inclusion criteria used to construct the dependent variable. The "cause" variable of the Brief IPQ was not entered into the regression analysis as patients could indicate multiple causes for their fatigue/CFS. Multicollinearity did not prove to be an issue. The linearity of the logit assumption was tested using the Box-Tidwell test. As physical activity (min/day) (PA) violated the linearity of the logit assumption, a categorical variable for PA was used based on the median. All other variables entered into the model were continuous. Table 2 shows the results of the logistic regression analysis.

The regression model was statistically signficant, $\chi^{2}(8$, $N=384)=165.98, p<.001)$, indicating that the model was able to distinguish between the two patient groups. The model explained between $35.1 \%\left(\mathrm{Cox} \&\right.$ Snell $\left.R^{2}\right)$ and $46.8 \%$ (Nagelkerke $R^{2}$ ) of the variance in the dependent variable and correctly classified $76.6 \%$ of the cases. Patients with higher consequences, timeline, and identity beliefs were more likely to be classified as having CFS. In contrast, persons with higher levels of PA and higher levels of "All or Nothing" behavior were less likely to be classified as having CFS.

\section{Discussion}

\section{Main Findings}

As expected, CFS patients reported higher levels of fatigue severity, a longer duration of fatigue, and more somatic complaints. 
Table 1 Descriptives of and differences between the CFS and fatigued non-CFS patients

\begin{tabular}{|c|c|c|c|c|c|c|}
\hline Variable & Non-CFS $N=192$ & CFS $N=192$ & Test for differences & $95 \% \mathrm{CI}^{\mathrm{a}}$ & $p$ & Effect size $r$ \\
\hline \multicolumn{7}{|l|}{ Patient characteristics } \\
\hline Age, years & $40.22 \pm 12.08$ & $40.50 \pm 12.20$ & $t=-.227$ & $-2.72,2.16$ & .821 & - \\
\hline Gender (female) & $170(88.5)$ & $170(88.5)$ & - & - & & \\
\hline Educational level (N, \%) & & & $\chi^{2}=4.63$ & - & .111 & - \\
\hline Primary education ${ }^{\mathrm{b}}$ & $1(0.5)$ & $7(3.6)$ & & & & \\
\hline Secondary education & $53(27.6)$ & $53(27.6)$ & & & & \\
\hline Higher education & $138(71.9)$ & $132(68.8)$ & & & & \\
\hline \multicolumn{7}{|l|}{ Fatigue and somatic complaints } \\
\hline Fatigue severity & $43.97 \pm 6.81$ & $47.83 \pm 7.31$ & $U=12176.5$ & - & .000 & -.29 \\
\hline Fatigue duration, weeks & $40.30 \pm 30.93$ & $454.85 \pm 459.52$ & $U=4393$ & - & .000 & -.66 \\
\hline Somatic complaints & $11.07 \pm 4.50$ & $14.23 \pm 4.54$ & $t=-6.86$ & $-4.07,2.26$ & .000 & -.33 \\
\hline \multicolumn{7}{|l|}{ Determinants } \\
\hline Average hours of sleep per night ${ }^{c}$ & $7.01 \pm 1.43$ & $7.55 \pm 1.81$ & $t=-3.25$ & $-0.87,-0.21$ & .001 & -.16 \\
\hline Minutes of PA per week & $221.68 \pm 304.67$ & $154.98 \pm 541.20$ & $U=12064.5$ & - & .000 & .30 \\
\hline \multicolumn{7}{|l|}{ Illness representations } \\
\hline Consequence & $6.93 \pm 1.73$ & $8.50 \pm 1.57$ & $U=8981.5$ & - & .000 & -.41 \\
\hline Timeline & $5.39 \pm 1.99$ & $7.57 \pm 1.95$ & $t=-10.86$ & $-2.58,-1.79$ & .000 & -.48 \\
\hline Personal control & $4.48 \pm 2.00$ & $4.13 \pm 2.13$ & $t=1.68$ & $-0.06,0.77$ & .094 & - \\
\hline Treatment control & $3.44 \pm 2.56$ & $4.47 \pm 2.58$ & $t=-3.95$ & $-1.55,-0.52$ & .000 & -.20 \\
\hline Identity & $5.33 \pm 2.24$ & $7.41 \pm 2.08$ & $U=8510$ & - & .000 & -.05 \\
\hline Understanding & $6.23 \pm 2.43$ & $5.71 \pm 2.65$ & $t=1.99$ & $0.01,1.03$ & .048 & - \\
\hline Emotional representation $^{\mathrm{c}}$ & $6.01 \pm 1.65$ & $6.34 \pm 2.02$ & $t=-1.74$ & $-1.40,-0.08$ & .083 & - \\
\hline \multicolumn{7}{|l|}{ Attributions of fatigue $(N, \%)$} \\
\hline A germ or virus & $35(18.2)$ & $120(62.5)$ & $\chi^{2}=78.16$ & - & .000 & -.45 \\
\hline Diet or eating habits & $75(39.1)$ & $63(32.8)$ & $\chi^{2}=1.63$ & - & .202 & - \\
\hline Pollution & $31(16.1)$ & $31(16.1)$ & $\chi^{2}=0.00$ & - & 1.00 & - \\
\hline Hereditary & $19(9.9)$ & $30(15.6)$ & $\chi^{2}=2.83$ & - & .092 & - \\
\hline Chance & $41(21.4)$ & $69(35.9)$ & $\chi^{2}=9.99$ & - & .002 & -.16 \\
\hline Stress & $160(83.3)$ & $128(66.7)$ & $\chi^{2}=14.22$ & - & .000 & .19 \\
\hline My own behavior & $105(54.7)$ & $65(33.9)$ & $\chi^{2}=16.89$ & - & .000 & .21 \\
\hline Others behavior & $85(44.3)$ & $58(30.2)$ & $\chi^{2}=8.12$ & - & .004 & - \\
\hline Poor medical care & $8(4.2)$ & $38(19.8)$ & $\chi^{2}=22.23$ & - & .000 & -.24 \\
\hline Limiting behavior $^{\mathrm{c}}$ & $18.88 \pm 4.58$ & $22.43 \pm 5.07$ & $t=-7.19$ & $-4.52,-2.58$ & .000 & -.34 \\
\hline All or nothing behavior & $16.59 \pm 5.61$ & $14.89 \pm 4.68$ & $t=3.17$ & $0.65,2.76$ & .002 & .16 \\
\hline Social support & $6.68 \pm 1.65$ & $6.60 \pm 1.83$ & $U=18388.5$ & - & .968 & - \\
\hline Depression & $6.83 \pm 5.29$ & $6.88 \pm 5.61$ & $U=18185.5$ & - & .820 & - \\
\hline Anxiety & $6.00 \pm 5.22$ & $6.09 \pm 5.26$ & $U=18241$ & - & .860 & - \\
\hline \multicolumn{7}{|l|}{ Outcome variables } \\
\hline Physical functioning & $58.09 \pm 19.00$ & $35.75 \pm 20.26$ & $t=11.15$ & $18.40,26.28$ & .000 & .49 \\
\hline Employment status $(N, \%)$ & & & $\chi^{2}=72.12$ & - & .000 & .27 \\
\hline Working, fulltime & $63(32.8)$ & $16(8.3)$ & & & & \\
\hline Working, part-time & $81(42.2)$ & $71(37.0)$ & & & & \\
\hline Not working & $27(14.1)$ & $96(50.0)$ & & & & \\
\hline Studying & $21(10.9)$ & $9(4.7)$ & & & & \\
\hline Medical visits, no. & $3.45 \pm 3.84$ & $4.49 \pm 4.60$ & $U=15145.5$ & - & .002 & -.16 \\
\hline
\end{tabular}

Values are the mean \pm SD unless otherwise indicated. Bonferroni corrected 0.05 alpha level 0.002

$P A$ physical activity

${ }^{\text {a }}$ Confidence interval of the difference

${ }^{\mathbf{b}}$ More than $20 \%$ of cells had expected count less than 5, an exact significance test was selected for Pearson's $\chi^{2}$

${ }^{\mathbf{c}}$ Levene's test of equal variance not assumed 
Table 2 Predictors of chronic fatigue syndrome $(N=384)$

\begin{tabular}{|c|c|c|c|c|c|c|}
\hline Predictors & $\beta$ & SE & Wald & OR & $95 \% \mathrm{Cl}^{\mathrm{b}}$ & $p$ \\
\hline Constant & -5.59 & 1.04 & 28.99 & .00 & & .000 \\
\hline Sleep & .043 & .084 & .268 & 1.044 & $0.89-1.23$ & .605 \\
\hline Min of PA per week (high) ${ }^{\mathrm{a} b}$ & -.744 & .270 & 7.610 & .475 & $0.28-0.81$ & .006 \\
\hline Consequence & .302 & .097 & 9.682 & 1.353 & $1.12-1.64$ & .002 \\
\hline Timeline & .428 & .071 & 36.426 & 1.530 & $1.34-1.76$ & .000 \\
\hline Treatment Control & .009 & .054 & .028 & 1.009 & $0.91-1.12$ & .868 \\
\hline Identity & .170 & .073 & 5.337 & 1.185 & $1.03-1.37$ & .021 \\
\hline All or nothing behavior & -.062 & .025 & 5.954 & .940 & $0.90-0.99$ & .015 \\
\hline Limiting behavior & .020 & .033 & .376 & 1.020 & 0.96-1.09 & .540 \\
\hline
\end{tabular}

CFS and fatigued non-CFS patients also differed in their causal attribution of fatigue. CFS patients more frequently attributed their fatigue to external and physical causes and non-CFS patients to internal causes. This is in line with the results of other studies $[12,13]$. Attribution of symptoms to physical causes has been previously associated with decreased physical activity and higher fatigue in CFS patients, suggesting that this attribution tendency is a maintaining factor [13].

In contrast with the study by Darbishire et al. [9] and Evengård et al. [11], we did not find differences in anxiety and depression between both groups. Differences in measurement instruments and study setting could partly explain this finding. The results of both previous studies could also be subject to a type 1 error, as no statistical correction for multiple comparisons was made.

As expected, CFS patients reported worse physical functioning and a lower employment rate than the fatigued nonCFS group. This is in line with other studies [9, 10]. As in the study by Darbishire et al. [9], CFS patients also paid significantly more visits to their GP and medical specialists.

The results of the multiple logistic regression analysis showed that patients who are more physically active are less likely to be classified as CFS. The amount of sleep per night was not a significant determinant. With regard to illness representations, the results showed that patients who believed that their fatigue was associated with more severe consequences, that it would last for a longer period of time, and patients who attributed more additional symptoms to their condition were more likely to be classified as CFS, a finding that is similar to other studies [9, 16]. Finally, patients who reported lower levels of "all or nothing behavior" were more likely to have a CFS diagnosis. This finding may suggest that "all-or-nothing" behavior is more characteristic of the earlier stages of fatigue, which is in line with a prospective cohort study [16], demonstrating that, within a patient cohort suffering from an acute viral infection, "all-or-nothing" behavior was a strong predictor of the development of CFS over time.

\section{Limitations and Strengths}

A limitation of this study is that the diagnosis of CFS was based on a self-report measure, which could have led to misclassification of some patients. Likewise, the presence of other somatic diseases and/or psychiatric disorders was not ruled out by means of clinical evaluations. The fact that both groups were for the most part recruited in a different way (patient organization for CFS versus primary health care centers for the non-CFS patients) may also have influenced the results. The present study included only quantity of sleep at night. Future studies should also take into account the amount of time spent sleeping during the day, as well as sleep quality, thus allowing for a more complete measurement of the sleep variable. In addition, other potentially important physical determinants such as BMI should also be taken into account in future studies.

Another limitation is the cross-sectional design of the study which does not allow for conclusions about the direction of significant associations. In the present study, there is, e.g., a significant difference in fatigue duration and severity between both study groups, which is, however, most likely due to the inclusion criteria used to distinguish between the CFS and the non-CFS group. In addition, other significant differences that were found (e.g., in physical activity and all-or-nothing behavior) are not necessarily precursors, but could also be consequences of being faced with chronic fatigue. For this reason, future prospective studies are needed to examine whether these factors are indeed risk factors for developing CFS.

An important strength of the present study, compared to other case control studies, is its sample size as well as the fact that the CFS and the fatigued non-CFS patients were matched by age and gender. In addition, this is the first study to include so many potential determinants of a distinction between both groups. 


\section{Conclusion}

CFS patients and fatigued non-CFS patients appear to differ in many respects. The differences found in this study with respect to physical, cognitive, and behavioral factors may point at potential risk factors for the transition from medically unexplained fatigue to CFS. A prospective study should explore the predictive value of these risk factors in order to develop a screening instrument that would allow for identifying primary care patients at risk at an early stage in view of the development of interventions.

Compliance with Ethical Standards All procedures followed were in accordance with the ethical standards of the responsible committee on human experimentation (institutional and national) and with the Helsinki Declaration of 1975, as revised in 2000. Informed consent was obtained from all patients for being included in the study.

Conflict of Interest The authors declare that they have no competing interests.

Open Access This article is distributed under the terms of the Creative Commons Attribution 4.0 International License (http:// creativecommons.org/licenses/by/4.0/), which permits unrestricted use, distribution, and reproduction in any medium, provided you give appropriate credit to the original author(s) and the source, provide a link to the Creative Commons license, and indicate if changes were made.

\section{References}

1. Krupp LB, Alvarez LA, LaRocca NG, Scheinberg LC. Fatigue in multiple sclerosis. Arch Neurol. 1988;45(4):435-7.

2. Lerdal A, Wahl A, Rustoen T, Hanestad BR, Moum T. Fatigue in the general population: a translation and test of the psychometric properties of the Norwegian version of the fatigue severity scale. Scand J Public Health Suppl. 2005;33(2):123-30. doi:10.1080/ 14034940410028406.

3. Pawlikowska T, Chalder T, Hirsch SR, Wallace P, Wright DJ, Wessely SC. Population based study of fatigue and psychological distress. BMJ. 1994;308(6931):763-6.

4. Ricci JA, Chee E, Lorandeau AL, Berger J. Fatigue in the U.S. workforce: prevalence and implications for lost productive work time. J Occup Environ Med. 2007;49(1):1-10. doi:10.1097/01. jom.0000249782.60321.2a.

5. van't Leven M, Zielhuis GA, van der Meer JW, Verbeek AL, Bleijenberg G. Fatigue and chronic fatigue syndrome-like complaints in the general population. Eur J Pub Health. 2010;20(3): 251-7. doi:10.1093/eurpub/ckp113.

6. Bleijenberg G. Chronic fatigue and chronic fatigue syndrome in the general population. Health Qual Life Outcomes. 2003;1:52. doi:10. 1186/1477-7525-1-52

7. Fukuda K, Straus SE, Hickie I, Sharpe MC, Dobbins JG, Komaroff A. The chronic fatigue syndrome: a comprehensive approach to its definition and study. International Chronic Fatigue Syndrome Study Group. Ann Intern Med. 1994;121(12):953-9.
8. Joyce J, Hotopf M, Wessely S. The prognosis of chronic fatigue and chronic fatigue syndrome: a systematic review. QJM. 1997;90(3): 223-33.

9. Darbishire L, Ridsdale L, Seed PT. Distinguishing patients with chronic fatigue from those with chronic fatigue syndrome: a diagnostic study in UK primary care. Br J Gen Pract. 2003;53(491): $441-5$.

10. Solomon L, Nisenbaum R, Reyes M, Papanicolaou DA, Reeves WC. Functional status of persons with chronic fatigue syndrome in the Wichita, Kansas, population. Health Qual Life Outcomes. 2003;1:48. doi:10.1186/1477-7525-1-48.

11. Evengård B, Jonzon E, Sandberg A, Theorell T, Lindh G. Differences between patients with chronic fatigue syndrome and with chronic fatigue at an infectious disease clinic in Stockholm. Sweden. Psychiatry Clin Neurosci. 2003;57(4):361-8.

12. Weinman J, Petrie KJ, Moss-morris R, Horne R. The illness perception questionnaire: a new method for assessing the cognitive representation of illness. Psychol Health. 1996;11(3):431-45. doi: 10.1080/08870449608400270.

13. Moss-Morris R. Symptom perceptions, illness beliefs and coping in chronic fatigue syndrome. J Ment Health. 2005;14(3):223-35. doi: 10.1080/09638230500136548.

14. Cairns R, Hotopf M. A systematic review describing the prognosis of chronic fatigue syndrome. Occup Med. 2005;55(1):20-31. doi: 10.1093/occmed/kqi013.

15. Hempel S, Chambers D, Bagnall AM, Forbes C. Risk factors for chronic fatigue syndrome/myalgic encephalomyelitis: a systematic scoping review of multiple predictor studies. Psychol Med. 2008;38(7):915-26. doi:10.1017/S0033291707001602.

16. Moss-Morris R, Spence MJ, Hou R. The pathway from glandular fever to chronic fatigue syndrome: can the cognitive behavioural model provide the map? Psychol Med. 2011;41(5):1099-107. doi: 10.1017/S003329171000139X.

17. Nijrolder $\mathrm{I}$, van der Horst $\mathrm{H}$, van der Windt $\mathrm{D}$. Prognosis of fatigue. A systematic review. J Psychosom Res. 2008;64(4):335-49. doi:10. 1016/j.jpsychores.2007.11.001.

18. Wagner D, Nisenbaum R, Heim C, Jones JF, Unger ER, Reeves WC. Psychometric properties of the CDC Symptom Inventory for assessment of chronic fatigue syndrome. Popul Health Metrics. 2005;3:8. doi:10.1186/1478-7954-3-8.

19. Beurskens AJ, Bultmann U, Kant I, Vercoulen JH, Bleijenberg G, Swaen GM. Fatigue among working people: validity of a questionnaire measure. Occup Environ Med. 2000;57(5):353-7.

20. Kroenke K, Spitzer RL, Williams JB. The PHQ-15: validity of a new measure for evaluating the severity of somatic symptoms. Psychosom Med. 2002;64(2):258-66.

21. Wendel-Vos GC, Schuit AJ, Saris WH, Kromhout D. Reproducibility and relative validity of the short questionnaire to assess health-enhancing physical activity. J Clin Epidemiol. 2003;56(12):1163-9.

22. Broadbent E, Petrie KJ, Main J, Weinman J. The brief illness perception questionnaire. J Psychosom Res. 2006;60(6):631-7. doi:10. 1016/j.jpsychores.2005.10.020.

23. Spence M, Moss-Morris R, Chalder T. The Behavioural Responses to Illness Questionnaire (BRIQ): a new predictive measure of medically unexplained symptoms following acute infection. Psychol Med. 2005;35(4):583-93.

24. Derogatis LR, Melisaratos N. The Brief Symptom Inventory: an introductory report. Psychol Med. 1983;13(3):595-605.

25. Ware Jr J, Kosinski M, Keller SD. A 12-Item Short-Form Health Survey: construction of scales and preliminary tests of reliability and validity. Med Care. 1996;34(3):220-33. 